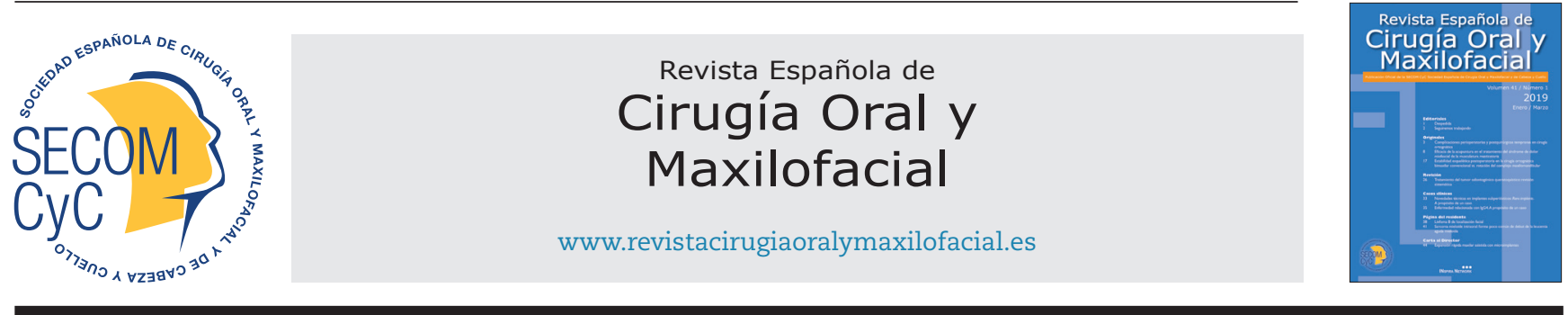

\title{
Original
}

\section{Tratamiento de las fracturas subcondíleas y de cuello de cóndilo mandibular mediante abordaje intraoral endoscópicamente asistido}

\author{
T. Creo Martínez, M. V. Núñez Vera, A. Tofé Povedano y J. A. Rodríguez Ruiz \\ d Especialista Facultativo de Área, Servicio de Cirugía oral y Maxilofacial, Hospital Puerta del Mar, Cádiz, España
}

INFORMACIÓN DEL ARTÍCULO

Historia del artículo:

Recibido el 10 de abril de 2018

Aceptado el 3 de julio de 2018

\section{Palabras clave:}

Fracturas de cóndilo, abordaje endoscópicamente guiado, oclusión, bloqueo intermaxilar.

\begin{abstract}
R E S U M E N
Introducción: El tratamiento de las fracturas de cóndilo sigue generando controversia, optándose en la mayoría de los casos por reducciones cerradas con tratamientos subóptimos. Presentamos nuestros resultados en 26 pacientes con fracturas subcondíleas o de cuello de cóndilo mandibular tratados mediante abordaje intraoral endoscópicamente asistido.

Material y método: Seguimiento prospectivo observacional de 26 pacientes que fueron atendidos en nuestro Servicio de Cirugía Oral y Maxilofacial del Hospital Universitario Virgen del Mar de Cádiz, desde marzo de 2010 hasta enero de 2018. De estos 26 pacientes, 23 fueron hombres y 3 mujeres, con una edad media de 29 años.

Resultados: De los 26 casos operados, 6 fueron fracturas de cuello y 20 fueron fracturas subcondíleas. Todos los pacientes salieron de quirófano con la boca abierta, una oclusión estable y buena apertura oral. La complicación más importante fue una infección que se resolvió con antibioterapia.

Discusión: Nuestro equipo ha seguido la filosofía de otros autores de abordar todas las fracturas de cóndilo, incluso aquellas con un desplazamiento menor de $10^{\circ}$, atendiendo a la premisa de que si no se bloquean otras fracturas no desplazadas mandibulares que previamente a la introducción de placas de osteosíntesis se bloqueaban, ¿por qué hemos de realizar esta práctica en las fracturas de cóndilo, con la consiguiente agresión a la articulación?

Conclusión: La reducción abierta con abordaje intraoral asistido por endoscopia constituye una técnica segura, reproducible y eficaz en la mayor parte de las fracturas extracapsulares.
\end{abstract}

${ }^{*}$ Autor para correspondencia

Correo electrónico: tesicreo@gmail.com (T. Creo Martínez).

DOI: 10.20986/recom.2019.1033/2019

1130-0558/@ 2019 SECOM. Publicado por Inspira Network. Este es un artículo Open Access bajo la licencia CC BY-NC-ND (http:// creativecommons.org/licenses/by-nc-nd/4.0/). 


\section{A B S T R A C T}

\section{Keywords:}

Condylar fractures, endoscopically guided approach, occlusion; intermaxillary block.
Introduction: The treatment of condylar fractures continues to generate controversy, opting in most cases for closed reductions with suboptimal treatment. We present our results in 26 patients treated by endoscopically assisted intraoral approach as treatment for subcondylar and neck condyle fractures.

Material and method: Prospective observational follow-up of 26 patients who were treated at our Oral and Maxillofacial Surgery Service at the Virgen del Mar University Hospital of Cádiz from March 2010 to January 2018. Of these 26 patients, 23 were men and 3 women, with an age average of 29 years.

Results: Of the 26 operated cases, 6 were neck fractures and 20 were subcondylar. All the patients came out of the operating room with their mouths open, a stable occlusion and good oral opening. The most important complication was an infection that resolved with antibiotic therapy.

Discussion: Our team has followed the philosophy of other authors to address all condylar fractures, even those with a displacement of less than $10^{\circ}$, following the premise that, if other non-displaced mandibular fractures are not blocked prior to the introduction of osteosynthesis plates were blocked, why should we perform this practice in the fractures of condyle with the consequent aggression to the joint?

Conclusion: Open reduction with endoscopic assisted intraoral approach is a safe, reproducible and effective technique in most extracapsular fractures.

\section{INTRODUCCIÓN}

En el tratamiento de las fracturas de mandíbula está comúnmente aceptado que el objetivo es conseguir una reducción anatómica y recuperar la funcionalidad lo antes posible, excepto en las fracturas de cóndilo mandibular. Las fracturas de cóndilo mandibular tienen una alta incidencia (35\% de todas las fracturas de mandíbula $)^{1} \mathrm{y}$ pueden producir discapacidad funcional grave. Su tratamiento correcto genera gran controversia.

En las fracturas de cóndilo de los niños se crea un remodelado restitucional con capacidad de volver a la normalidad. Una actuación sobre el cóndilo puede afectar gravemente al crecimiento, por lo que en ellos el tratamiento conservador de fracturas dislocadas produce resultados funcionales satisfactorios a largo plazo, a pesar de la alta frecuencia de aberraciones radiológicas ${ }^{2}$. En los adultos y niños a partir de los 14 años el remodelado es funcional y, por tanto, la capacidad de adaptarse es menos predecible y la necesidad de reducción abierta es mayor. En la mayoría de los casos se opta por la reducción cerrada con bloqueo intermaxilar (BIM) alámbrico o elástico, porque la reducción de las fracturas de cóndilo abiertas es una intervención compleja y delicada, y puede ser técnicamente muy difícil reducir y fijar los segmentos. Además, el abordaje abierto tiene riesgos anatómicos y entre sus posibles complicaciones se incluye lesión del nervio facial, cicatrices, hemorragia y compromiso vascular del segmento proximal, mientras que los pacientes a los que se les realiza BIM suelen tener resultados aceptables ${ }^{3}$.

Sin embargo, el tratamiento cerrado de las fracturas subcondíleas es más propenso a resultados subóptimos (particularmente, desviación con la apertura oral, pérdida de altura de la rama y maloclusión) ${ }^{4}$, además de que se produce una inmovilización ciega de los fragmentos con un BIM prolongado, con el problema que ello supone para la dinámica de la articulación temporomandibular, lo que implica una rehabi- litación funcional y oclusal prolongada con mayor riesgo de artrosis por inmovilización.

Clásicamente, el problema de la reducción abierta era su dificultad y el riesgo de complicaciones ${ }^{5}$. Hoy en día esto no es un problema gracias al abordaje intraoral endoscópicamente asistido. Su finalidad es evitar abordajes extraorales y sus posibles complicaciones, está indicado en fracturas extracapsulares, luxadas o no, tiene bajo riesgo y la recuperación funcional es inmediata ${ }^{6}$.

En este artículo presentamos nuestros resultados en 26 pacientes tratados de fractura extracapsular de cóndilo mediante abordaje intraoral endoscópicamente asistido.

\section{MATERIAL Y MÉTODO}

Presentamos un seguimiento prospectivo observacional de 26 pacientes que fueron atendidos en nuestro Servicio de Cirugía Oral y Maxilofacial del Hospital Universitario Virgen del Mar de Cádiz desde marzo de 2010 hasta enero de 2018. De estos 26 pacientes, 23 fueron hombres y 3 mujeres, con una edad media de 29 años.

A todos los pacientes se les realizó, como valoración preoperatoria al ingreso, una ortopantomografía y una tomografía computarizada facial con reconstrucción 3D de forma rutinaria, seleccionándose para la cirugía aquellos pacientes que padecían fracturas de cuello y subcondíleas según la clasificación de Ellis ${ }^{7}$, y que presentaban desde fracturas mínimamente desplazadas hasta fracturas altamente desplazadas según la clasificación de $\mathrm{Bhagol}^{8}$. Se excluyeron de la cirugía aquellos pacientes edéntulos o con un estado de salud general previo muy limitado que no se iban a beneficiar de la ventaja de salir de quirófano con la boca abierta, como grandes discapacitados o pacientes encamados de forma crónica. Tratamos mediante reducción abierta tanto a pacientes con fracturas de cóndilo 
aisladas como a aquellos pacientes que presentaban fracturas de cóndilo asociadas a otras fracturas faciales, y especialmente indicada en pacientes que sufrían fractura conminuta mandibular, donde la reducción de los cóndilos nos ha ayudado a mantener la altura de la mandíbula.

Todos los pacientes fueron informados mediante un consentimiento informado de las opciones terapéuticas y de las ventajas e inconvenientes de cada una.

Realizamos un seguimiento a la semana, 4 semanas, 12 semanas y 24 semanas, registrando en la historia clínica las siguientes variables: a) máxima apertura oral, b) laterodesviación, c) acortamiento de la rama mandibular respecto a la contralateral en casos unilaterales, d) dolor a la apertura y e) oclusión.

Realizamos por sistema una ortopantomografía postoperatoria y a los 3 meses, salvo en los pacientes politraumatizados, en los que tuvimos que realizar una tomografía computarizada de control por su dificultad para la movilidad. Con las pruebas de imagen evaluamos la alineación de los fragmentos y la remodelación ósea.

En la cirugía empleamos material quirúrgico específico para la reducción de fracturas condíleas asistida por endoscopia: óptica de $4 \mathrm{~mm}$ y $30^{\circ}$ de Karl Storz-Endoskope ${ }^{\circledR}$, con una vaina de retracción endoscópica de Synthes ${ }^{\circledR}$ y un periostótomo largo aspirador de Synthes ${ }^{\circledR}$, un separador de Langenbeck invertido y un separador de rama, además de una pinza Bengolea para manejar la placa de osteosíntesis. La osteosíntesis se realizó con placas lambda y trapezoidales de Synthes ${ }^{\circledR}$ que se colocaron con un sistema de perforación-destornillador de contraángulo con pestaña móvil que sujeta la placa.

Todas las cirugías fueron realizadas por un cirujano principal y un ayudante. Se empleó anestesia general e intubación nasotraqueal. Se intervinieron en primer lugar todas las fracturas faciales asociadas, dejando para el final las fracturas de cóndilo.
Inicialmente se lleva al paciente a oclusión, que se mantiene con tornillos de IMF y alambre. Se aborda la fractura mediante incisión sobre la línea oblicua mandibular y disección subperióstica y se exponen el trazo de fractura y el fragmento condíleo. Se introduce el endoscopio para ver la posición de los fragmentos y el estado de la fractura (Figura 1), se destensan los alambres y se procede a la reducción de la fractura (Figura 2). Una vez conseguida, se tensa otra vez el alambre y se procede a la fijación de los fragmentos (Figura 3). Una vez fijados, se retira el bloqueo alámbrico. El paciente es dado de alta a las 48 horas tras realizar un control de imagen (Figura 4).

Los objetivos de la cirugía fueron conseguir la reducción anatómica de los fragmentos y fijarlos mediante osteosíntesis para que el paciente saliese con la boca abierta.

\section{RESULTADOS}

De los 26 casos operados, 6 fueron fracturas de cuello y 20 fracturas fueron subcondíleas.

De los 26 pacientes, 2 pacientes tenían fractura de cóndilo en el contexto de fracturas complejas, 6 pacientes presentaban otras fracturas de mandíbula asociadas, 3 pacientes fracturas de cóndilo asociadas con otras fracturas faciales, 4 pacientes fracturas subcondíleas bilaterales y 11 pacientes fractura de cóndilo unilateral.

El tiempo medio quirúrgico para las fracturas de cóndilo fue de 120 minutos (el tiempo máximo fue de 450 minutos en un paciente con fractura de cóndilo bilateral asociada a otras fracturas faciales) y de 90 minutos para las fracturas condíleas unilaterales poco desplazadas. En todos los casos se realizó la osteosíntesis, y todos los pacientes salieron con la boca abierta y $\sin$ bloqueo.

En la ortopantomografía de control postoperatorio se consideró que el alineamiento de la fractura estaba corregido en

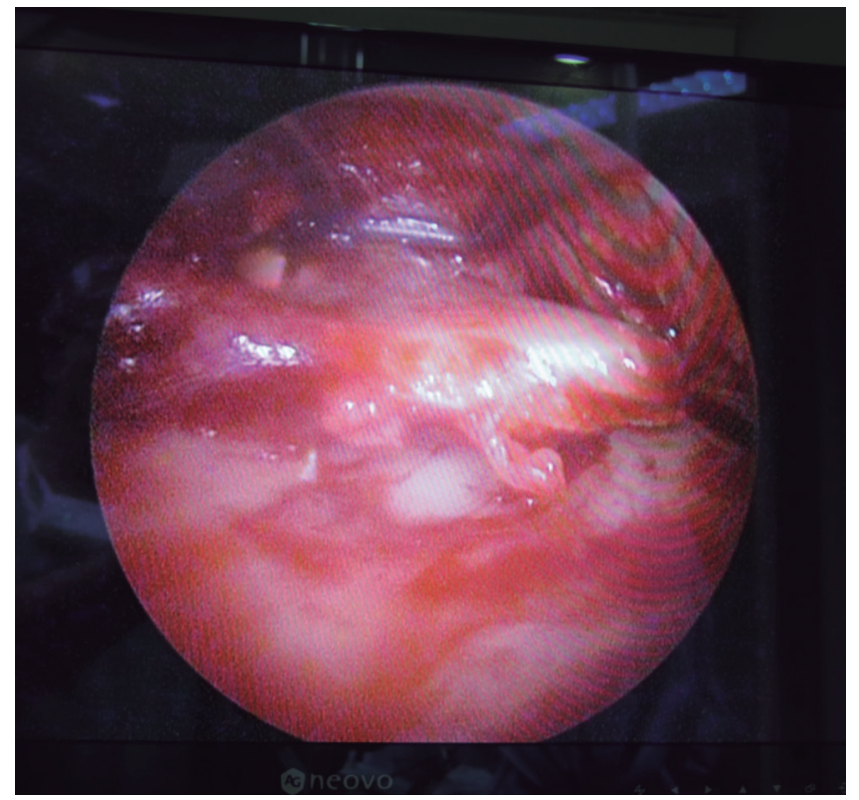

Figura 1. Exploración de los fragmentos de la fractura.

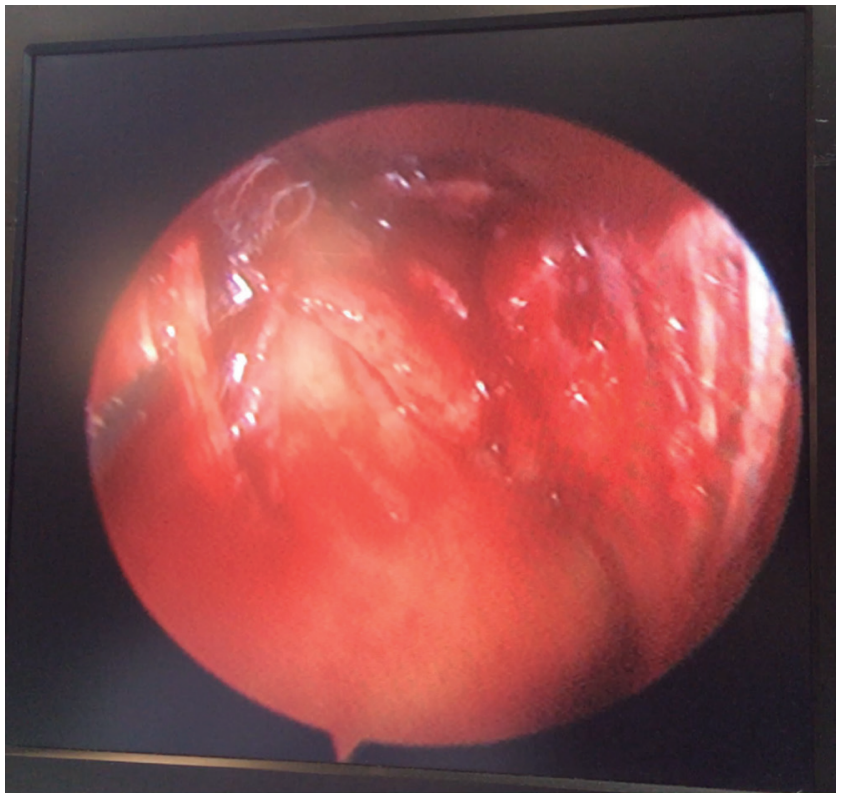

Figura 2. Reducción anatómica de los fragmentos. 


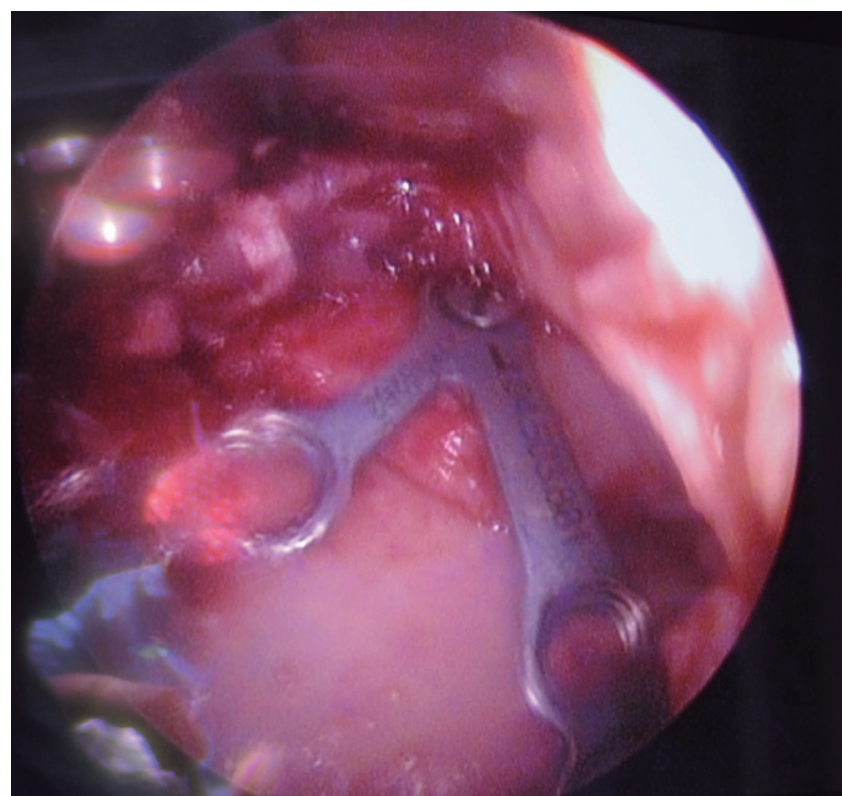

Figura 3. Fijación de los fragmentos con osteosíntesis.

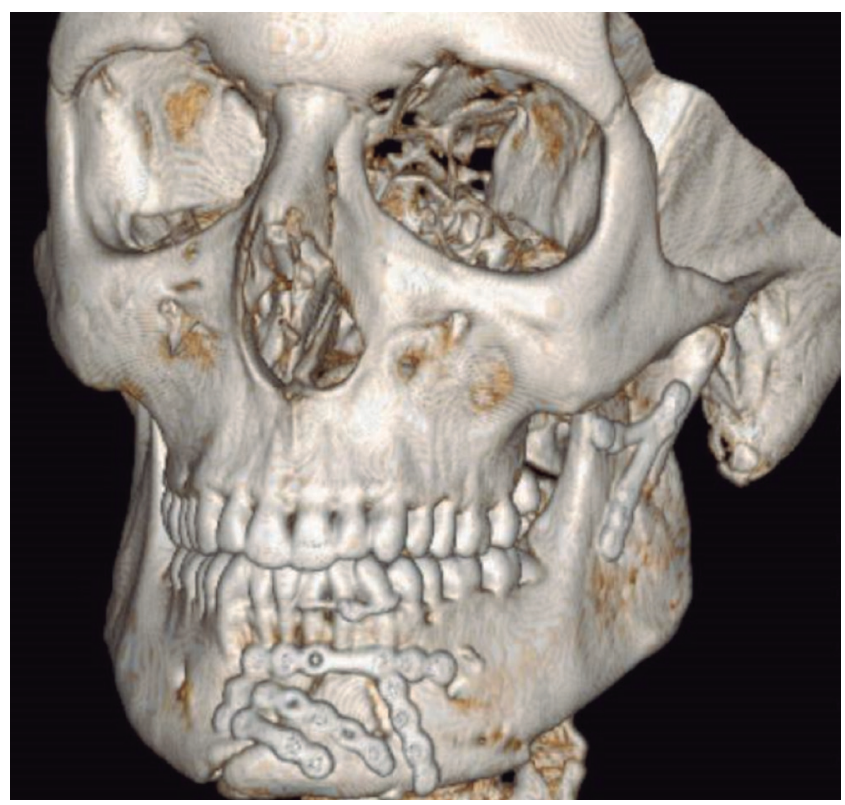

Figura 4. Control radiográfico de la fractura.

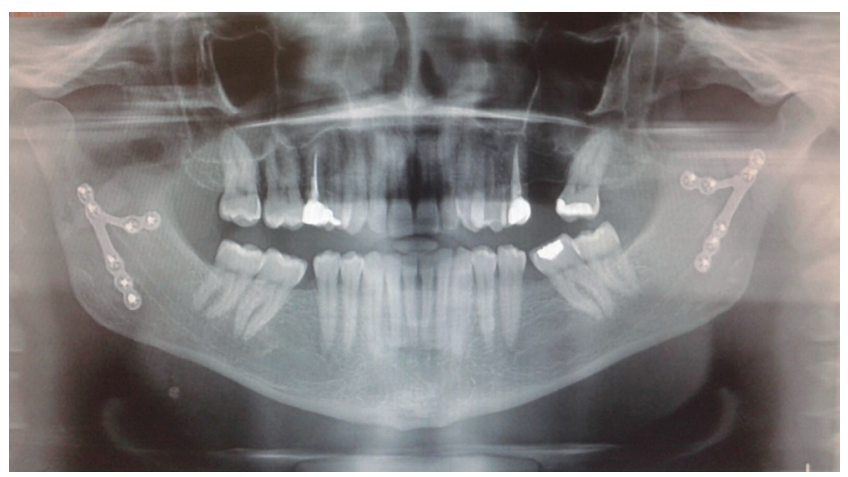

Figura 5. Control radiográfico al alta.
$21(80,8 \%)$, mejorado en $4(15,4 \%)$ e igual en 1 (3,8 \%), que se mantuvo con dieta triturada durante 1 mes y control estrecho para controlar la oclusión. Todos ellos mantuvieron una oclusión igual a la previa, consiguiendo una apertura de $35 \mathrm{~mm}$ en 4 semanas. En todos ellos logramos mantener la altura de la rama mandibular, sin mordida abierta posterior en caso de los bilaterales, y solo tuvimos un paciente con laterodesviación a la apertura, que se mantuvo al alta.

Se produjo una infección postoperatoria, con absceso de la zona, que se drenó y no hubo que retirar la osteosíntesis. Un paciente presentó alteraciones en la sensibilidad de la zona de forma transitoria, y dos pacientes necesitaron tratamiento con miorrelajantes por dolor durante un tiempo a la apertura oral en relación con la musculatura masticatoria.

No tuvimos complicaciones con la rama frontoorbitaria del facial ni hubo, durante este tiempo, pérdida de la osteosíntesis.

Todos los pacientes salieron de quirófano con la boca abierta, una oclusión estable y buena apertura oral. Fueron dados de alta a los 6 meses (Figura 5).

\section{DISCUSIÓN}

Aunque clásicamente se indicaba para todas las fracturas de cóndilo un bloqueo intermaxilar alámbrico $(\mathrm{BIM})^{9}$, hay autores que, indicando el mismo tipo de tratamiento, ya hablan de resultados desfavorables en un porcentaje significativo de pacientes tratados con esta técnica, como son Marker y cols. ${ }^{10}$, que observaron que un $10 \%$ de los pacientes presentan una máxima apertura oral reducida.

En 2007, Eulert y cols. ${ }^{11}$ publicaron las complicaciones que se evidenciaban a largo plazo con el tratamiento conservador, tras seguir a 1812 pacientes tratados con BIM o abordaje de fractura de cóndilo. En el grupo tratado con tratamiento conservador, un 28,6 \% de los pacientes presentaron maloclusión a los 3 años y un $64 \%$ la presentaron a los 10 años, por lo que se evidenció que había diferencias estadísticas significativas entre las fracturas tratadas de forma conservadora con BIM y las que se reducían con osteosíntesis. Igualmente, Ellis ${ }^{4}$ publicó un mayor porcentaje de maloclusión tras tratamiento conservador significativamente superior que el observado con la reducción y fijación de la fractura condílea.

Pero estos autores que observaban los problemas con el BIM, eran reticentes al abordaje de las fracturas de cóndilo, porque este se tenía que realizar extraoral, con los problemas y secuelas consecuentes a ello, como la paresia facial en un 17,2 \% de los casos y el 7,5 \% de los casos de cicatrices hipertróficas ${ }^{4}$.

Estas reticencias al abordaje de las fracturas condíleas desaparece con el abordaje intraoral, cuyas primeras series fueron publicadas en 1998 por Lee y cols. ${ }^{12}$, apoyado por un transbucal para realizar la osteosíntesis, con excelentes resultados. Posteriormente, otros autores ${ }^{13,14}$ se sumaron a la realización de esta técnica, pero el cambio real se produjo cuando en 2005 Schön y cols. ${ }^{15}$ publicaron una serie amplia en la que realizaron abordaje intraoral asistido por endoscopia y osteosíntesis con contraángulo en todas las fracturas de cóndilo, incluso en aquellas con gran luxación y desplazamiento, con muy buenos resultados. De este modo, las reticencias al abordaje de este tipo de fracturas debidas a las posibles complicaciones del abordaje extraoral desaparecen con el abordaje intraoral, con el que, tras una curva 
de aprendizaje, se puede resolver la gran mayoría de fracturas de cuello y subcondíleas, independientemente del desplazamiento ${ }^{6}$.

En 2012, Kokemueller y cols. ${ }^{16}$ realizaron un estudio intercentro para valorar las diferencias entre abordaje transoral con reducción abierta y reducción cerrada, en un estudio prospectivo de 75 pacientes con fracturas de cóndilo desplazadas al menos $30^{\circ}$. En estos pacientes se procedió a la reducción y osteosíntesis de estas fracturas mediante abordaje transoral, y se obtuvieron excelentes resultados sin grandes complicaciones. Por consiguiente, el abordaje intraoral asistido por endoscopia se postula como la técnica ideal para el tratamiento de cualquier tipo de fractura de cóndilo.

La introducción de esta nueva técnica con visión en dos dimensiones y un espacio reducido para la manipulación conlleva una curva de aprendizaje más o menos prolongada para el cirujano ${ }^{17}$, con un consumo de tiempo importante hasta que el se acostumbra a esta nueva forma de trabajar, pero los resultados tanto a corto como largo plazo y el poco riesgo de secuelas justifican esta curva importante de aprendizaje.

En 2016, You y cols..$^{18}$ publicaron una serie de 50 casos de pacientes operados de fractura de cóndilo mediante abordaje endoscópico (usado para todo tipo de fracturas de cóndilo, incluso en las que clásicamente se indicaba BIM) y presentaron unos excelentes resultados, sin reabsorciones condilares y con recuperación funcional y estructural excelente, sin lesiones del facial, hipoestesia transitoria ni por supuesto cicatrices visibles, por lo que recomiendan el uso del abordaje transoral endoscópicamente asistido en todos los casos.

Nosotros, en nuestra serie, hemos tratado mediante reducción y fijación todo tipo de fracturas de cóndilo, incluso aquellas que para ciertos autores clásicamente debían tratarse con BIM, porque consideramos que mejora la calidad el hecho de que el paciente se vaya con la boca abierta, y disminuye el trauma sobre la articulación temporomandibular que supone un BIM prolongado, además de obtenerse magníficos resultados. Por otro lado, también hemos abordado fracturas de cóndilo luxadas que provocarían acortamiento de la rama mandibular u otras secuelas importantes.

Nuestro equipo ha seguido la filosofía de You ${ }^{18}$ o Goizue$\mathrm{ta}^{17}$ de abordar todas las fracturas de cóndilo, incluso aquellas con un desplazamiento menor de $10^{\circ}$, atendiendo a la premisa de que, si no se bloquean otras fracturas no desplazadas mandibulares que previamente a la introducción de placas de osteosíntesis se bloqueaban, ¿por qué hemos de realizar esta práctica en las fracturas de cóndilo, con la consiguiente agresión a la articulación?

En nuestro trabajo todos los pacientes salieron con buena apertura oral, que fue mejorando progresivamente a lo largo de los días, sin complicaciones importantes y con oclusión estable y mantenida a lo largo del tiempo.

\section{CONCLUSIÓN}

La reducción abierta con abordaje intraoral asistido por endoscopia constituye una técnica segura, reproducible y eficaz en la mayor parte de las fracturas extracapsulares.

Es necesario que el cirujano maxilofacial aumente su experiencia y adiestramiento en el uso de esta técnica para reducir las complicaciones de este tipo de fracturas.
Se debe intentar equiparar la osteosíntesis de estas fracturas por vía intraoral a las de otras zonas de la mandíbula, independientemente del grado de desplazamiento o luxación que presenten.

\section{B I B L I O G R A F Í A}

1. Ellis E, Throckmorton GS. Treatment of mandibular condylar process fractures: Biological considerations. J Oral Maxillofac Surg. 2005;63:115-34.

2. Chori J, Oh N, Kim IK. A follow-up study of condyle fracture in children. Int J Oral Maxillofac Surg. 2005;34:851-8.

3. Cascone P, Spallaccia F, Fatone FM, Rivaroli A, Saltarel A, Iannetti G. Rigid versus semirigid fixation for condylar fracture: experience with the external fixation system. J Oral Maxillofac Surg. 2008;66:265-71.

4. Ellis E, Simon P, Throckmorton GS. Occlusal results after open and closed treatment of fracture of the mandibular condylar process. J Oral Maxillofac Surg. 2000;58:260-8.

5. Zide MF. Open reduction of mandibular condyle fractures. Indications and technique. Clin Plast Surg. 1989;16(1):69-76.

6. Schoen R, Fakler O, Metzger MC, Weyer N, Schmelzeisen R. Preliminary functional results of endoscope-assisted transoral treatment of displaced bilateral condylar mandible fractures. Int J Oral Maxillofac Surg. 2008;37:111-6.

7. Ellis E, Simon P, Throckmorton G. Occlusal results after open and closed treatment of fracture of the mandibular condylar process. J Oral Maxillofac Surg. 2000;58:260-8.

8. Bhagol A, Singh V, Kumar I, Verma A. Prospective evaluation of a new classification system for the management of mandibular subcondylar fractures. J Oral Maxillofac Surg. 2011;69:1159-65.

9. Blevins C, Gores RJ. Fractures of the mandibular condyloid process: results of conservative treatment in 140 patients. J Oral Surg Anesth Hos Dent Service. 1961;19:28.

10. Marker P, Nielsen A, Bastian HL. Fractures of the mandibular chondyle. Part 2: Results of treatment of 348 patients. Br J Oral Maxillofac Surg. 2000;38:422-6.

11. Eulert S, Proff P, Bokan I, Blens T, Gedrange T, Reuther J, et al. Study on treatment of condylar process fractures of the mandible. Ann Anat. 2007;189:377-83.

12. Lee C, Mueller RV, Lee K, Mathes SJ. Endoscopic subcondylar repair: functional, aesthetic, and radiographic outcomes. Past Reconst Surg. 1998;102:1434-43.

13. Chen CT, Lai JP, Tung TC, Chen YR. Endoscopically assisted mandibular subcondylar fracture repair. Plast Reconstr Surg. 1999;103:60-5.

14. Lauer G, Schmelzeisen R. Endoscope-assisted fixation of mandibular condylar process fractures. J Oral Maxillofac Surg. 1999;57:36-9.

15. Schön R, Fakler O, Gellrich NC, Schmelzeisen R. Five-year experience with the transoral endoscopically assisted treatment of displaced condylar mandible fractures. Plast Reconstr Surg. 2005;116:44-59.

16. Kokemueller H, Konstantinovic VS, Barth EL, Goldhahn S, von See C, Tavassol F, et al. Endoscope-assisted transoral reduction and internal fixation versus closed treatment of mandibular condylar process fractures- a prospective double-center study. J Oral Maxillofac Surg. 2012;70:384-95.

17. Goizueta-Adame CC, Pastor-Zuazaga D, Agüero-de Dios E, Sebastián-López C. Osteosíntesis intraoral asistida por endoscopia en las fracturas del proceso condilar de la mandíbula: revisión de 53 casos. Rev Esp Cir Oral Maxilofac. 2012;34(4):156-65.

18. You HJ, Moon KC, Yoon ES, Lee BI, Park SH. Clinical and radiological outcomes of transoral endoscope-assisted treatment of mandibular condylar fractures. Int J of Oral and Maxillofac Surg. 2016;45(3):284-91. 